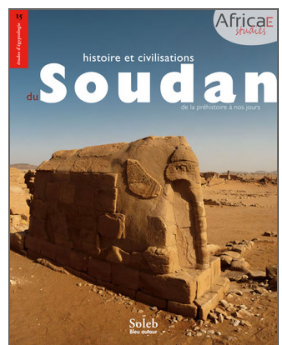

Olivier Cabon (dir.)

Histoire et civilisation du Soudan

De la préhistoire à nos jours

Africae

\title{
9. Batailles sur les ruines de Méroé
}

Blemmyes et Nubiens : les royaumes postméroïtiques. 350-543 apr. J.-C.

\section{Claude Rilly}

DOI : $10.4000 /$ books.africae. 2827

Éditeur: Africae, Soleb, Bleu autour

Lieu d'édition : Paris, Khartoum

Année d'édition : 2017

Date de mise en ligne : 17 janvier 2022

Collection : Africae Studies

EAN électronique : 9782493207074

\section{OpenEdition}

\section{Books}

http://books.openedition.org

\section{Référence électronique}

RILLY, Claude. 9. Batailles sur les ruines de Méroé: Blemmyes et Nubiens : les royaumes postméroïtiques.

350-543 apr. J.-C In : Histoire et civilisation du Soudan : De la préhistoire à nos jours [en ligne]. Paris,

Khartoum : Africae, 2017 (généré le 28 janvier 2022). Disponible sur Internet : <http://

books.openedition.org/africae/2827>. ISBN : 9782493207074. DOI : https://doi.org/10.4000/

books africae. 2827 


\section{batailles sur \\ les ruines de Méroé}

Blemmyes et Nubiens:

les royaumes postméroïtiques

350-543 apr. J.-C. 
La période qui suit la chute du royaume de Méroé et s'étend jusqu'à la conversion officielle au christianisme des États qui ont pris son relais constitue deux siècles que l'on qualifie souvent d' "obscurs", entre 350 et 550 de notre ère. Les sources historiques qui les éclairent quelque peu ne concernent en effet que la Basse-Nubie. Il s'agit de textes d'historiens grecs ou de documents locaux, généralement aussi en grec. À la lecture de ces témoignages, trois acteurs se partageaient alors le pouvoir sur la région: les Blemmyes et les Noubades (ou Nobades) sur le terrain et, depuis son territoire en deçà de la première cataracte, auquel s'ajoutait la petite principauté autour des temples de Philae, l'Empire romain, dont la principale préoccupation était de contrer les raids que lançaient régulièrement les Blemmyes et les Noubades sur les villes du sud de la Haute-Égypte. Les Méroïtes ne sont plus cités comme une entité politique, alors qu’ils constituaient certainement la masse de la population.

Les sources archéologiques ne fournissent également que des informations partielles. Aucun complexe cultuel n'a été retrouvé jusqu’ici et très peu d'habitat. On connaît surtout des sites funéraires, qui marquent à la fois une rupture et une continuité par rapport aux enterrements méroïtiques. La forme pyramidale est abandonnée au profit du tumulus qui, particulièrement dans le sud du royaume koushite, n'avait jamais été totalement évincé; la position contractée pour les corps des défunts, devenue très rare en raison de l'utilisation de cercueils à l'imitation de l'Égypte, refait son apparition; les textes funéraires disparaissent. En revanche, y compris dans les enterrements des princes noubas, le rite funéraire, comprenant des libations et des sacrifices, est conservé; le matériel inhumé avec le défunt est très proche de celui des tombes méroïtiques et il ne fait pas de doute que tout ou partie de la religion funéraire koushite est adopté par les nouveaux arrivants. On observe au nord une recrudescence des «morts d'accompagnement», c'est-à-dire de personnes sacrifiées pour accompagner les princes dans l'Au-delà. Les grands absents de l'archéologie funéraire sont en revanche les Blemmyes, dont les chefs ne peuvent être associés de manière certaine avec aucun des cimetières retrouvés jusqu'à présent. Les pionniers de l'archéologie postméroïtique, notamment Reisner puis les Britanniques W. B. Emery et L. P. Kirwan, qui fouillèrent les tombes princières de Qustul et Ballaña en Basse-Nubie, ne pouvant décider de l'ethnie des défunts dans les sépultures qu'ils excavèrent, employèrent la désignation commode de "Groupe X» pour cette culture tardive, un terme que l'on retrouve encore utilisé dans certains travaux.

La continuité culturelle entre le méroïtique et la période suivante a amené plusieurs spécialistes, au premier rang desquels l'archéologue français Patrice Lenoble, à s’interroger sur la réalité de la "fin de Méroé»: 


\section{le Soudan, de la chute de Méroé au royaume Fung}

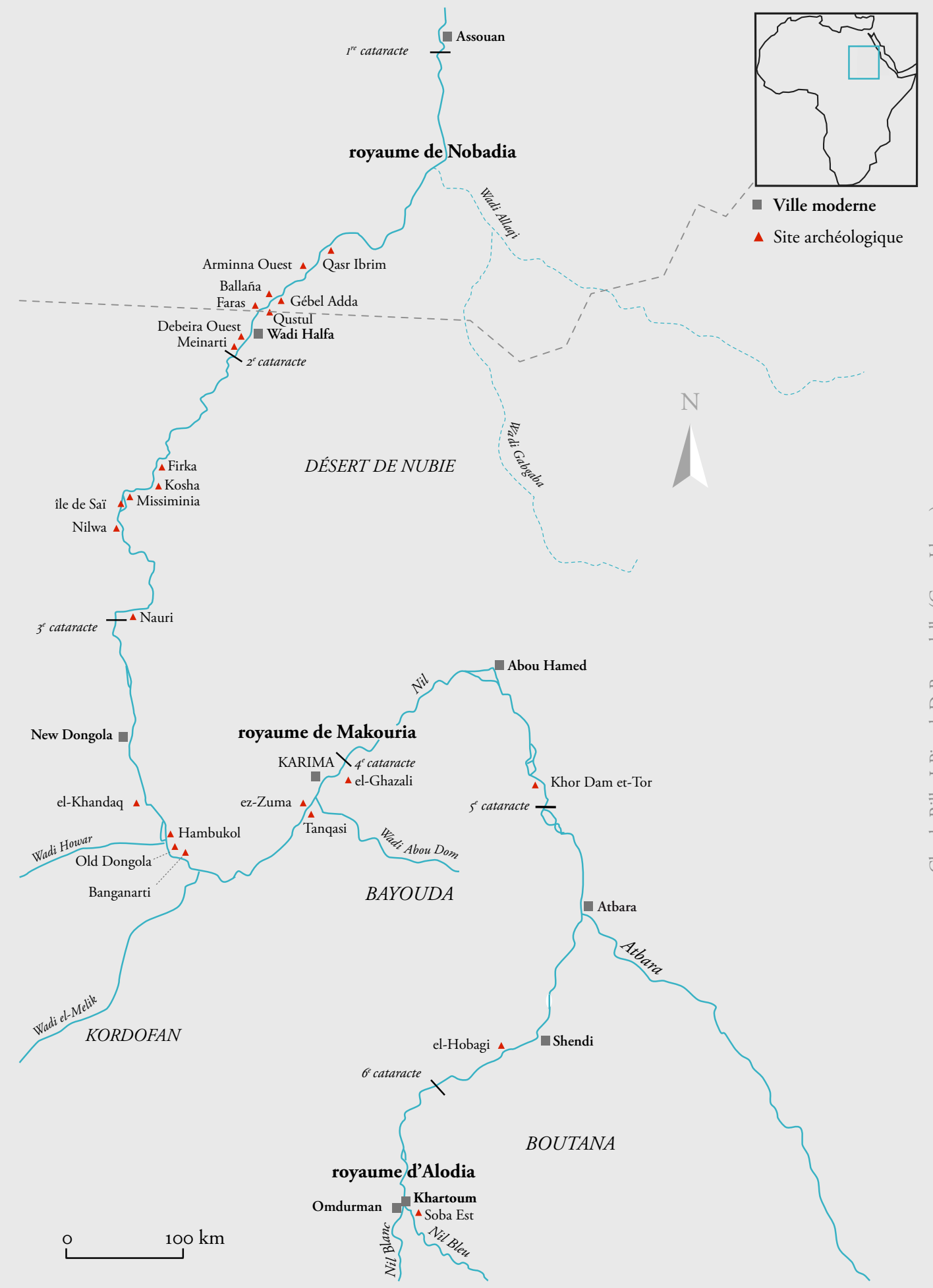


ne pouvait-on pas considérer que la civilisation méroïtique avait perduré au-delà de la chute de la capitale, sous de nouveaux monarques issus des tribus désormais maîtresses du territoire? Cette hypothèse s'accompagnait d'une réinterprétation de l'État méroïtique comme un Empire composé de plusieurs royaumes et dont le qore, "souverain», aurait été l'empereur. Cette thèse, qui s'appuyait sur trop peu d'éléments, est maintenant quasiment abandonnée. Le terme de "méroïtique postpyramidal», forgé par Lenoble pour entériner cette continuité et se substituer à celui de "Groupe X», a fait place aujourd'hui de manière presque unanime à l'appellation «postméroïtique».

\section{Les nouveaux maîtres de la Basse-Nubie : Blemmyes et Noubades}

Les nouveaux venus sur les rives du fleuve ne sont pas des inconnus: nous les avons croisés à maintes reprises dans les chapitres précédents, car ils ont, depuis des siècles, compté au nombre des adversaires des souverains napatéens et méroïtiques.

Les Blemmyes étaient un peuple couchitique ancien apparenté aux actuels Bedjas, une population originellement nomade, aujourd'hui séparée en plusieurs groupes depuis le sud de l'Égypte jusqu'en Érythrée, mais principalement à l'est du Soudan. Leur première mention se trouve dans la stèle que le roi napatéen Anlamani fit ériger à Kawa à la fin du $\mathrm{VII}^{\mathrm{e}}$ siècle av. J.-C., où il relate sa campagne militaire contre les Bulahau (Bw-l-hj-jw). Un siècle plus tard, vers 513, apparaît en Haute-Égypte, dans le papyrus démotique Rylands Ix, le terme Blhlm, "Blemmye», qui qualifie un chef de la police au service du vizir. Son rang élevé dans la hiérarchie montre l'intégration dans le royaume pharaonique de familles d'immigrants blemmyes, peut-être réfugiées en Égypte à la suite des campagnes napatéennes. Son nom égyptien, Ouahibrê-Merenrê, qui n'est autre que le nom de couronnement de Psammétique ${ }^{\text {Ir }}$ (664-610), est sans doute un hommage au roi qui accueillit ses aïeux. Par la suite, les Blemmyes sont cités dans les textes égyptiens sous diverses variantes: Blhm, Blhw.

Dans les sources napatéennes, ils semblent disparaître après le règne d'Anlamani mais il est très probable, comme nous l'avons proposé dans nos sections consacrées aux rois Harsiotef (voir p. 175 sq.) et Nastasen (voir p.179 sq.) (Iv siècle av. J.-C.), que le terme de Lehleh(es) ou Rehreh(es) qui désigne les plus dangereux de leurs adversaires est un nom péjoratif donné par les Koushites au même peuple. Dans les sources grecques, le mot "Blemmyes» apparaît au $\mathrm{III}^{\mathrm{e}}$ siècle: selon le géographe 
alexandrin Ératosthène, ils vivent entre le Nil et la mer Rouge et sont vassaux des rois koushites. Ils sont voisins des Troglodytes (sans doute les ancêtres des Bedjas proprement dits), qui habitent plus près des côtes. Par la suite, il semble que certains géographes gréco-romains aient confondu Blemmyes et Troglodytes. Ainsi, pour Pline l'Ancien, les Blemmyes sont des êtres étranges qui ne possèdent pas de tête et ont les yeux et la bouche fixés sur le thorax. Mais il mentionne bien, entre Nil et mer Rouge, les Troglodytes comme des êtres humains normaux, sinon qu'ils sont d'une incroyable vitesse à la course à pied et parlent par cris, une caractéristique que Pline a empruntée à Hérodote.

Les Blemmyes disposaient bien entendu d'une véritable langue. En 2003, le philologue Gerald Browne, spécialiste du vieux-nubien, publia aux États-Unis sur cet idiome disparu un ouvrage singulier d'une trentaine de pages. Il le rédigea en effet en latin, tout en le faisant paraître aux presses de son université d'Urbana-Champaign dans l'Illinois sous le titre Textus blemmyicus aetatis Christianae ("Un texte blemmye d'époque chrétienne»). La préface commence par cette adresse ironique: «Ô lecteur très avisé et, comme je le crains, très rare, tu tiens entre tes mains l'opuscule qui contient, pour autant que je sache, tout ce qui reste de la langue blemmye." Il y étudiait à nouveau un ostracon médiéval retrouvé à Saqqara en Égypte par Quibell en 19071908 et que Griffith tenait pour l'unique témoignage interne, rédigé en lettres coptes, de la langue blemmye. Browne démontrait qu'il contenait une paraphrase des Psaumes, ce qui prouvait qu'au moins une partie des Blemmyes avait alors adopté la foi chrétienne. Il y ajoutait l'analyse des quelques noms de princes et de notables blemmyes qui sont parvenus jusqu'à nous. La comparaison qu'il effectuait avec la langue bedja actuelle montrait une très grande proximité. Si les Blemmyes n'étaient pas les ancêtres directs des Bedjas, ils constituaient en tout cas une branche extrêmement proche.

Il ne fait pas de doute que les Méroïtes, comme les rois napatéens, aient eu à en découdre avec les Blemmyes. Un passage des Panégyriques latins, daté des alentours de l'an 291 de notre ère, décrit des combats acharnés entre les deux camps. Toutefois, on ignore par quel terme, apparemment non transparent, sont désignés les Blemmyes dans les textes méroïtiques, si bien qu'il est pour l'heure impossible de les repérer dans les récits de campagnes militaires des souverains de Méroé. Du côté de l'Empire romain, les chroniqueurs semblent indiquer assez tôt des conflits récurrents avec les Blemmyes en Haute-Égypte, mais les termes utilisés pour les désigner, souvent "Barbares» ou "Indiens» (sic!), sont imprécis. Il faut attendre le règne de l'empereur Probus pour que soit signalée explicitement une victoire romaine contre les Blemmyes autour de la ville de Coptos en 280. En 336, Constantin reçoit à Byzance des délégations 
blemmyes et "éthiopiennes", ces dernières constituant peut-être une des ultimes représentations à l'étranger du royaume méroïtique moribond. La chute finale de Méroé va permettre aux Blemmyes de s'installer au bord du fleuve jusqu'au début du $\mathrm{v}^{\mathrm{e}}$ siècle.

Les Noubades, dont le nom est attesté sous de nombreuses variantes (Nobades, Nobatai, Annoubades, etc.), sont la frange la plus septentrionale des Noubas, un peuple que nous avons longuement présenté dans les chapitres précédents (voir «le long règne de Harsiotef», p.175-179, et «Les incursions des Noubas et l'expansion d'Axoum», p. 314-318). La stèle du roi napatéen Nastasen, au IV ${ }^{\mathrm{e}}$ siècle av. J.-C. (voir p.179-181), décrit deux campagnes contre les Noubas, désignés non par ce terme péjoratif qui signifie «esclave» en méroïtique, mais par leur nom «neutre» Makha (méroïtique $M h o$, protonubien *magur ou *magi). La première expédition oppose l'armée napatéenne aux Makha de Dakana, l'autre à ceux de Sharakha. Cette différenciation entre deux lieux qui ne sont apparemment pas contigus est confirmée au siècle suivant par le géographe grec Ératosthène. Dans sa description de l' «Éthiopie», il signale que les Noubas, alors installés dans l'ouest du Soudan, sont divisés en plusieurs principautés.

Dans sa stèle de victoire, le roi d'Axoum Ézana relate ses combats contre les Noubas, désignés comme «Noirs» au début du texte, dans le sud du royaume de Méroé désormais morcelé. Mais il signale que ses troupes ont fait une incursion sur le territoire des «Noubas rouges». Or, c'est par cet adjectif que les Abyssins des hauts plateaux, résultat d'un mélange entre immigrés sabéens et population locale, se qualifient traditionnellement, par opposition aux «Noirs", terme qui désigne les tribus indigènes non métissées. On sait qu'au $\mathrm{vI}^{\mathrm{e}}$ siècle les royaumes nubiens du Nil sont au nombre de trois, Nobadia au nord, Makouria au centre et Alodia (ou Alwa) au sud. Les Noubas «noirs» sont très certainement les fondateurs du royaume nubien d'Alodia, frontalier avec l'Abyssinie. Quant aux «Noubas rouges", il pourrait s'agir des Noubades, si l'on admet que l'installation des Makourites dans la boucle du Nil s'est effectuée plus tard. Toutefois, les chefferies noubas installées sur le fleuve à la période postméroïtique étaient plus nombreuses que les trois royaumes en place au vi siècle, si l'on en juge par les nécropoles princières connues: Qustul-Ballaña et Gemmai en BasseNubie, Firka et Kosha en Moyenne Nubie, ez-Zuma et Tanqasi à proximité de l'ancienne Napata, el-Hobagi face à Méroé.

Les Noubades forment en effet un groupe très particulier au sein des peuples nubiens. Alors que tous les autres parlers de cette famille présentent un vocabulaire de base très proche, la langue héritée des Noubades, appelée vieux-nubien au Moyen Âge et nobiin aujourd'hui, comporte des termes complètement différents qui empêchent encore actuellement 
le Soudan

\section{0}

des origines

à la chute

du sultanat

Fung leurs locuteurs de se comprendre par exemple avec les gens de Dongola, un peu plus au sud. Sans entrer ici dans les détails techniques, on peut montrer que cette particularité provient d'une situation originelle où un groupe de langue nubienne a formé une confédération avec une tribu d'une autre langue, celle-ci étant d'ailleurs apparentée au nara d'Érythrée et donc de manière lointaine au nubien et au méroïtique. Où et quand s'est formée cette confédération, on l'ignore, mais probablement avant l'installation sur les rives du Nil. Le nom de «Noubades» (et ses variantes) est bien entendu tiré de "Nouba», et il n'est pas le terme indigène, même s'il a fini par être adopté par les Noubades eux-mêmes dans leurs textes rédigés en grec, comme l'inscription du roi Silko que nous aborderons un peu plus loin. Le nom qu'ils se donnaient est en vieux-nubien Migi et provient d'un protonubien *magi qui, avec sa variante *magur, est l'autonyme des peuples nubiens.

Ce n'est qu'à partir du début $\mathrm{du}^{\mathrm{e}}$ siècle que les sources grécoromaines attestent la présence des Noubades en Basse-Nubie et leurs attaques sur les villes de Basse-Égypte. Mais il est possible que certaines mentions antérieures d' "Éthiopiens» en conflit avec le pouvoir romain, comme lors d'un raid contre Assouan vers 395 raconté dans L'histoire des moines d'Égypte, aient en fait désigné les Noubades plutôt que les Blemmyes, dont le nom était bien connu. Les sources méroïtiques de Basse-Nubie, essentiellement de courts passages biographiques des épitaphes, évoquent régulièrement des combats contre les Nouba (méroïtique $N o b$ ) ou les Makhu (Mho), depuis la table d'offrandes du vice-roi de Nubie Khalalakharora, au début de l'ère chrétienne, jusqu'à la fin de la période méroïtique. Nous avons vu également que, dans les dernières décennies du royaume de Méroé, deux épitaphes de Faras et du Gébel Adda mentionnent des liens diplomatiques entre les Méroïtes et un État "makhu», dont il est malheureusement impossible de préciser la localisation mais qui est sans doute limitrophe de la Basse-Nubie, voire constitué d'une portion de territoire déjà soustraite au pouvoir méroïtique. Le $\mathrm{v}^{\mathrm{e}}$ siècle verra les Noubades imposer une domination sans partage sur l'ensemble de la région et en expulser les Blemmyes.

\section{Rome face à de nouveaux voisins tumultueux}

On se souvient qu'en 298 l'empereur Dioclétien retira ses légions du Dodécaschène, le nord de la Basse-Nubie, conservant juste une garnison près de Philae pour en protéger les sanctuaires. Un accord fut sans aucun doute conclu avec Méroé pour qu'il prenne le relais de Rome dans la région libérée de la tutelle romaine, bien que les sources dont nous disposons soient confuses sur ce point. Mais à peine quelques décennies plus tard, 
le royaume méroïtique s'effondra et avec lui la sécurité qu'il pouvait assurer à la frontière avec l'Égypte. Les incursions des Blemmyes, puis des Noubades, sur les villes du sud de l'Égypte se multiplièrent, visant particulièrement les églises, les monastères et leurs trésors. Plusieurs pièces d'orfèvrerie chrétienne, dont un reliquaire d'argent, ont été retrouvées dans les hypogées des princes noubades inhumés à Ballaña et proviennent certainement de tels pillages. Les Blemmyes, quant à eux, étendirent leur rayon d'action très loin dans l'Égypte romaine puisque vers 371 est signalée une attaque contre l'oasis de Kharga et en 373 contre un monastère du Sinaï. Les prêtres d'Isis à Philae, dont le culte était encore toléré malgré l'irrésistible expansion du christianisme, durent renoncer pendant deux ans à effectuer le pèlerinage de la barque portant l'effigie de la déesse jusqu'à l'île proche de Biggeh, où elle était censée retrouver son divin époux Osiris. L'un des témoignages de ces exactions les plus poignants, bien qu'empli de déférence byzantine, nous a été conservé dans la supplique qu'un évêque de Syène (Assouan), Appion, adressa entre 425 et 450 aux empereurs d'Orient Théodose II et d'Occident Valentinien III, pour obtenir que la garnison de Philae étendît sa protection à son diocèse tout proche: 《 Je me trouve avec mes églises au milieu de barbares diaboliques, entre les Blemmyes et les Annoubades [= Noubades]; nous souffrons d'eux d'innombrables attaques, comme venues de l'invisible, sans qu'aucun soldat ne défende nos lieux. Puisque les églises placées sous mon autorité sont humiliées et qu'elles ne peuvent protéger ne serait-ce que ceux qui fuient ces tribus, je me jette et me roule à terre devant les divines et pures empreintes de vos pas pour que vous jugiez bon de décréter que nos saintes églises soient gardées par les soldats postés près de nous. \\ (Papyrus Leyde Z SB XX 14606, 5-9, texte grec établi par T. Eide, Fontes Historiae Nubiorum III [314].)

À de nombreuses reprises, les Romains tentèrent de remédier à cette situation selon leur politique habituelle vis-à-vis des peuples barbares qui harcelaient leurs frontières: des expéditions punitives, suivies de négociations pour établir un pacte de non-agression, voire un traité qui les reconnaissait comme alliés (foederati) de Rome. Mais la plupart du temps, ces conciliations restèrent sans lendemain, les exactions reprenant très vite comme avant. L'historien grec Priscus de Panium relate un tel épisode qui met en scène le duc de la Thébaïde Maximin vers 452. Après avoir défait les Blemmyes et les Noubades, Maximin entama avec leurs chefs tribaux des pourparlers sur l'île de Philae. Ils acceptèrent une trêve réduite dans un premier temps au mandat de Maximin sur la Thébaïde, dans un second à la durée de sa vie, puis, sur l'insistance du duc, à une période de cent ans. Des otages pris parmi les fils des chefs furent livrés aux Romains. En retour, ceux-ci accorderaient à leurs adversaires le libre accès aux temples de Philae 
le Soudan

\section{2}

des origines

à la chute

du sultanat

Fung

et permettraient à nouveau l'acheminement annuel de la statue de la déesse Isis jusqu'à leurs sanctuaires, afin qu'elle puisse servir pour les oracles. Mais, dès l'année suivante, Maximin vint à mourir, et aussitôt les Blemmyes et les Noubades dénoncèrent le traité et reprirent leurs otages par la force.

\section{Talmis (Kalabcha), capitale des Blemmyes sur le Nil}

Vers 394, le père de l'Église Épiphane de Salamine composa un Traité des douze gemmes où il décrivait et commentait les pierres précieuses qui auraient orné le pectoral du grand-prêtre Aaron, frère de Moïse. L'une d'elle offre à Épiphane l'occasion de parler des mines d'émeraudes, situées dans le désert Arabique, entre Nil et mer Rouge, non loin de Talmis (l'actuel Kalabcha). Il signale que cette cité, qui était auparavant sous la tutelle des Romains, vient de passer sous la domination des Blemmyes, ainsi que plusieurs autres. Elle y restera jusqu'aux victoires du roi noubade Silko vers 450 .

Kalabcha, une cité située à près de $60 \mathrm{~km}$ au sud d'Assouan, abritait un temple de grande dimension, commencé sous les Ptolémées et les rois mérö̈tiques, mais dont l'état actuel date essentiellement du règne d'Auguste. Il était consacré à un dieu local, Mandoulis, considéré comme un fils d'Isis. Son nom peut être écrit aussi bien avec un «d» qu'un « $\mathrm{r}$ " (démotique $M r$ ' et var.), ce qui laisse supposer qu'il comportait un /d/ rétroflexe (sonnant comme un « $\mathrm{r}$ ) présent aussi bien en mérö̈tique qu'en bedja actuel, langue apparentée au parler ancien des Blemmyes. Toutefois, cette divinité n'est pas attestée dans les textes méroïtiques et, bien que cette hypothèse ait été contestée par plusieurs spécialistes, il ne nous paraît pas impossible que Mandoulis ait été dès l'origine un dieu blemmye. Il est en tout cas certain qu'après la prise de Talmis il fit l'objet de la part des chefs et de la population blemmye d'un culte tout particulier, attesté notamment par une inscription grecque qui détaille l'organisation de congrégations religieuses en l'honneur de Mandoulis et de divinités inconnues par ailleurs, Abéné et Khopan.

D'autres inscriptions en grec témoignent à Kalabcha de la dévotion des rois blemmyes Tamal et Isemné, lequel cite un autre monarque, Dégou, comme prédécesseur. Il n'est toutefois pas certain que ces rois aient régné depuis Talmis. S’ils sont bien désignés par le titre de basileus, "roi», le texte mentionné précédemment sur l'organisation des congrégations indique que Talmis était gouverné non directement par le roi des Blemmyes mais par un "chef de tribu» (phylarkhos). Il répondait alors au nom de Phonen et ne devint roi que quelques années plus tard, comme nous le verrons plus loin dans sa lettre au roi noubade Abourni. Dans cette missive, son fils Breitek portera alors le titre de phylarkhos, ce qui laisse supposer que ces «chefs 


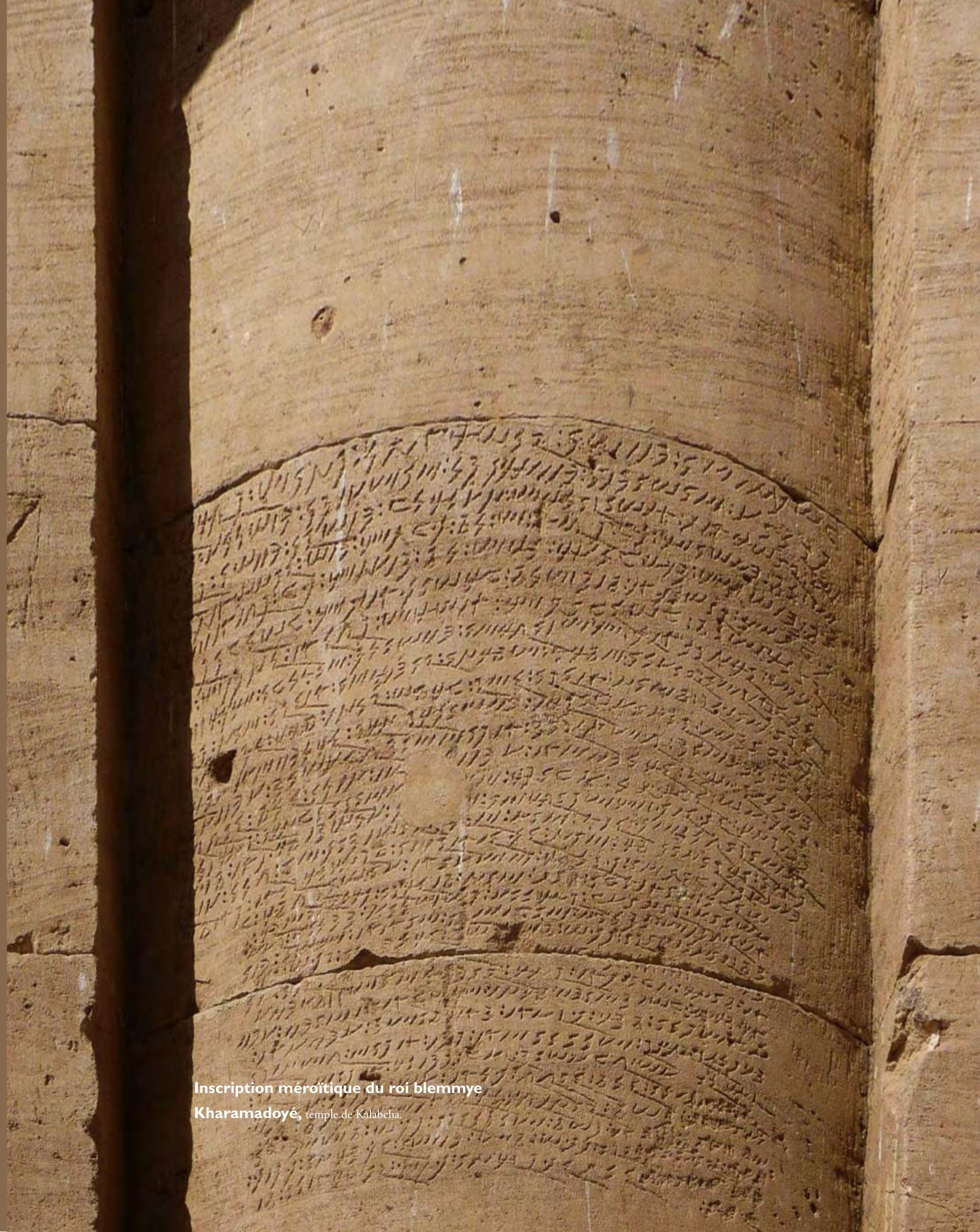


de tribu» étaient, au moins pour certains d'entre eux, des princes de sang nommés à ces postes par le souverain, dont la résidence était sans doute plus à l'est, sur les terres traditionnellement tenues par les Blemmyes.

Le temple de Kalabcha comporte également une inscription très fameuse, cette fois rédigée en méroïtique sur une colonne et constituant la dernière des chroniques de règne connues dans cette langue. Elle commémore un «roi» (méroïtique qore) nommé Kharamadoyé. Abondamment commentée depuis sa publication par Griffith en 1912, elle a été tour à tour attribuée à un souverain méroïtique, blemmye ou noubade et datée en conséquence du $\mathrm{IV}^{\mathrm{e}}$ ou du $\mathrm{V}^{\mathrm{e}}$ siècle. Bien qu'étudiée à deux reprises (1973 et 2003) par le méroïtisant Nicholas B. Millet, elle reste majoritairement incompréhensible. Tout au plus peut-on signaler qu'elle traite pour l'essentiel de la répartition du territoire de la Basse-Nubie au terme de plusieurs campagnes militaires. Parmi les peuples rivaux, on relève les Temey et les Mho, c'est-à-dire respectivement les Romains (anciennement Tmey, lit. «les Blancs") et les Noubades, cités côte à côte.

Millet avait noté la similitude entre un personnage nommé dans le texte Yisemeniye et le roi blemmye Isemné commémoré dans une inscription grecque du même temple. Il envisageait que ce souverain ait pu être l'adversaire principal de Kharamadoyé, qui aurait en conséquence été un roi noubade. S'il ne fait pas de doute que Yisemeniye est une version «méroïtisée» d'Isemné, rien n'indique, dans le contexte des deux passages où il est cité, qu'il ait été opposé à Kharamadoyé. Bien au contraire, il est qualifié de qore $l h$, "grand roi", "souverain suprême». À un autre endroit, des origines à la chute du sultanat Fung il est question des "huit rois du nord" (qore 8 hre-se) sur un territoire inclus entre Adere (lieu inconnu au sud) et Philae. Il nous paraît donc que, dans cette inscription, qore est l'équivalent du grec phylarkhos et qore-lh celui du grec basileus. Kharamadoyé, comme plus tard Phonen, aurait donc été un phylarkhos blemmye établi à Kalabcha, placé sous la suzeraineté du roi Isemné. Comme plusieurs spécialistes l'ont déjà relevé, son nom commence par l'élément khara qui désigne "dieu» en langue blemmye et est attesté dans d'autres noms de princes de cette ethnie.

Il reste à comprendre pour quelle raison un "chef de tribu » blemmye, au début $\mathrm{du} \mathrm{v}^{\mathrm{e}}$ siècle, a ressenti le besoin de faire graver à Kalabcha une inscription commémorative en méroïtique et non en grec, comme les rois blemmyes Tamal et Isemné, ou plus tard le roi noubade Silko. La rédaction du texte a été effectuée par un scribe méroïte, à une époque très tardive pour cette langue, comme en témoignent non seulement la paléographie mais aussi certaines orthographes inédites. Le long protocole initial qui détaille les titres du qore a été simplement recopié sur un modèle ancien, dont nous possédons des bribes par un fragment de stèle royale retrouvé 
à Qasr Ibrim et datant de la fin du III $^{\mathrm{e}}$ siècle de notre ère. Il est assez vraisemblable que le choix de la vieille langue de Koush, de préférence au grec, la lingua franca de l'Empire romain d'Orient et des pays adjacents, procède d'une volonté de présenter la domination blemmye sur Kalabcha comme une continuation du défunt royaume de Méroé auprès d'une population locale qui devait être encore en majorité composée de Méroïtes. Mais on ignore de quel poids ces derniers, qui désormais n'étaient plus maîtres de leur destin, pouvaient peser dans la rivalité qui opposait les nouveaux seigneurs de la Nubie, Blemmyes et Noubades.

\section{La victoire finale des rois noubades}

Un dernier texte gravé sur les murs du temple de Mandoulis, à l'entrée de la salle hypostyle, revêt une importance historique de premier plan. Il s'agit de l'inscription triomphale du roi noubade Silko. Contrairement à celle de Kharamadoyé, elle est rédigée en grec et non en méroïtique, notamment parce qu'elle devait pouvoir être lue des Blemmyes, qui utilisaient le grec dans leurs rares écrits. Elle est toutefois accompagnée de deux représentations gravées du souverain qui le placent dans la continuité des rois de Méroé. La première est empreinte d'influence gréco-romaine, dont n'étaient pas exemptes certaines figures de rois méroïtiques. Silko est monté sur un fringant destrier et transperce de sa lance un ennemi couché. Une Victoire ailée ajuste sur sa tête la couronne hemhem traditionnelle pour les monarques koushites et les pharaons de la période tardive, mais portée aussi par le dieu Mandoulis, maître de Kalabcha. La seconde figure du roi est presque entièrement égyptienne: Silko y est représenté debout, coiffé de la double couronne pharaonique avec uraus et corne d'Amon, tenant un sceptre et la croix de vie. L'inscription grecque prend place entre ces différents portraits du souverain.

Le texte a sans doute été composé par un scribe égyptien de BasseNubie frotté de culture grecque, car il comporte de nombreuses fautes où transparaît sa langue maternelle copte, tout en utilisant à l'occasion des termes rares ou recherchés. Il est possible que ce scribe ait été chrétien, car «le dieu» (ho theos) est au singulier, les divinités des Blemmyes par lesquelles ils prêtent serment sont désignées par le mot grec eidola, «idoles», et la double comparaison du souverain avec un lion et un ours, animal inconnu en Afrique orientale, a des parallèles dans la Bible. Mais cela ne signifie pas que Silko lui-même ait été chrétien, ce qui parait fort improbable et est de toute façon contredit par sa représentation en pharaon.

L'inscription reste, malgréses imperfections, compréhensible, contrairementà d'autres documentsgrecs de Nubie plusou moins contemporains. Silko s'y présente comme le roi des Noubades, sa propre ethnie, et plus largement 


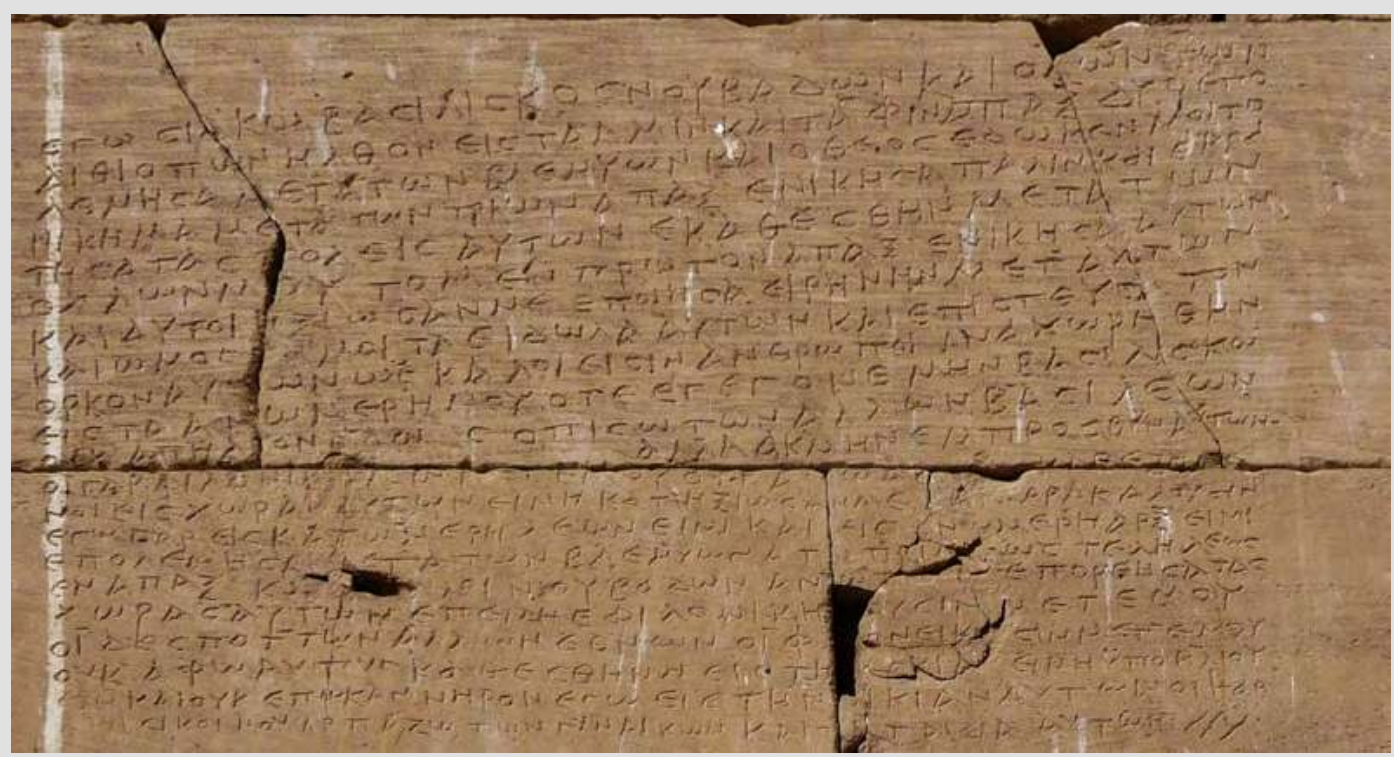

Inscription triomphale du roi Silko, gravée en grec sur un mur

du temple de Kalabcha, Basse-Nubie, milieu du Ve siècle.

\section{6}

des origines

à la chute

du sultanat

Fung
Moi, Silko, roi des Noubades et de tous les Éthiopiens, je suis venu à Talmis [Kalabcha] et Taphis [Tafa]. Par deux fois, j'ai guerroyé contre les Blemmyes et le dieu m'a donné la victoire. La troisième fois, j'ai vaincu à nouveau et me suis emparé de leurs cités; je m’y suis installé avec mes armées. La première fois, je les ai vaincus et eux-mêmes m’ont demandé [mes conditions]. J'ai fait la paix avec eux, ils m'ont juré par leurs idoles et j'ai cru à leur serment — qu'ils étaient des gens honnêtes et je me suis retiré vers mes terres en amont.

Lorsque je suis devenu roi, je ne suis pas du tout parti derrière les autres rois, mais juste à leur tête, car ceux qui me défient, je ne permets pas qu'ils restent dans leur royaume sans qu'ils demandent à traiter avec moi et requièrent mon jugement.

Je suis en effet dans les régions d'aval un lion et, dans les régions d'amont, un ours. J'ai guerroyé contre les Blemmyes depuis Primis (Qasr Ibrim) jusqu'à Télélis (Shellâl?); quant aux autres [tribus], en amont des Noubades, j'ai même une fois ravagé leurs terres, après qu'elles m'eurent défié. Quant aux chefs des autres peuples qui me défient, je ne permets pas qu'ils s'assoient à l'ombre, mais au dehors au soleil, au lieu de boire de [l'eau] fraîche à l'intérieur, dans leur maison. Quant à mes adversaires, j'enlève leurs femmes et leurs enfants. 


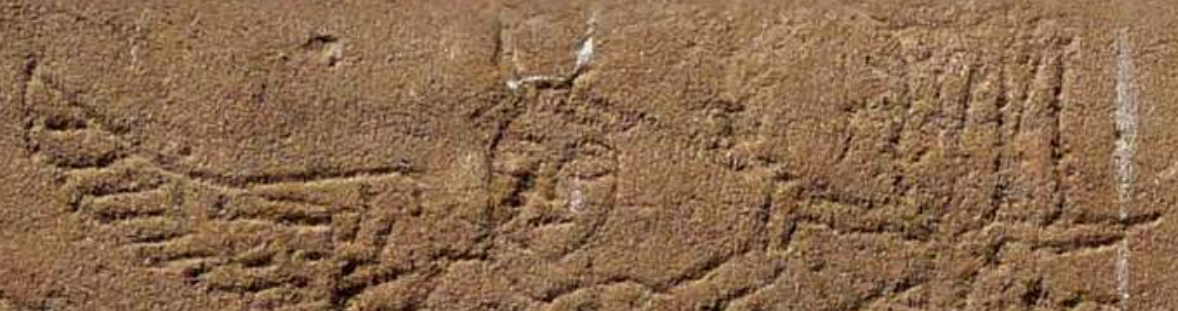

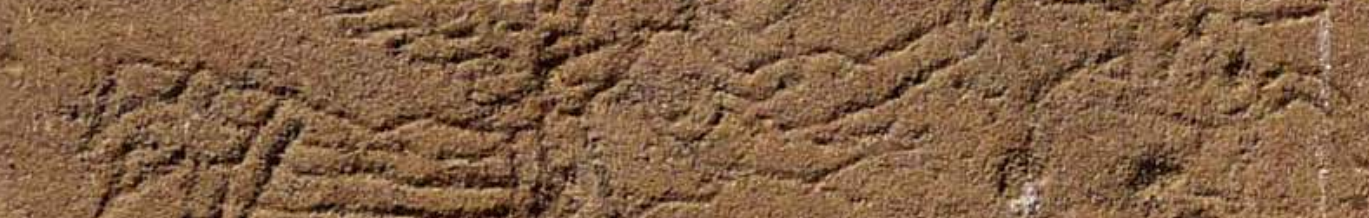

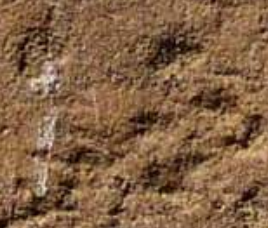

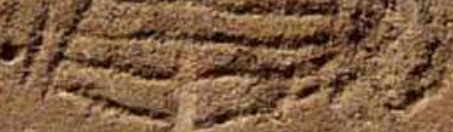

3
3

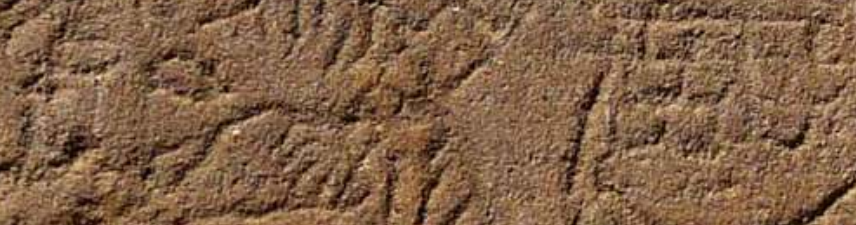

30.
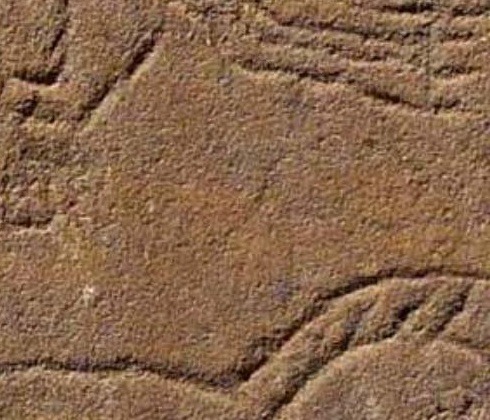

c)

t)

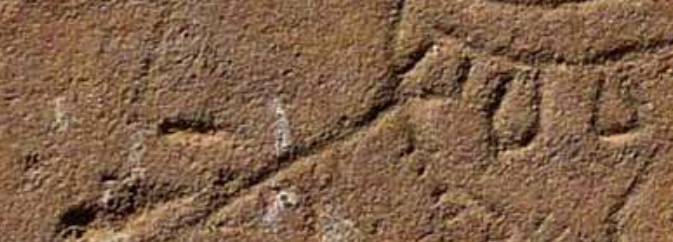

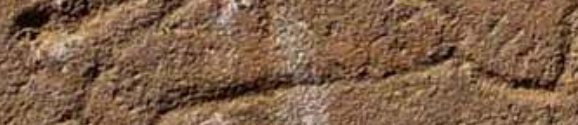

1. 1. fis

sep

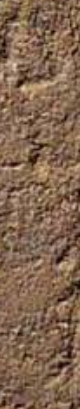

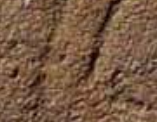
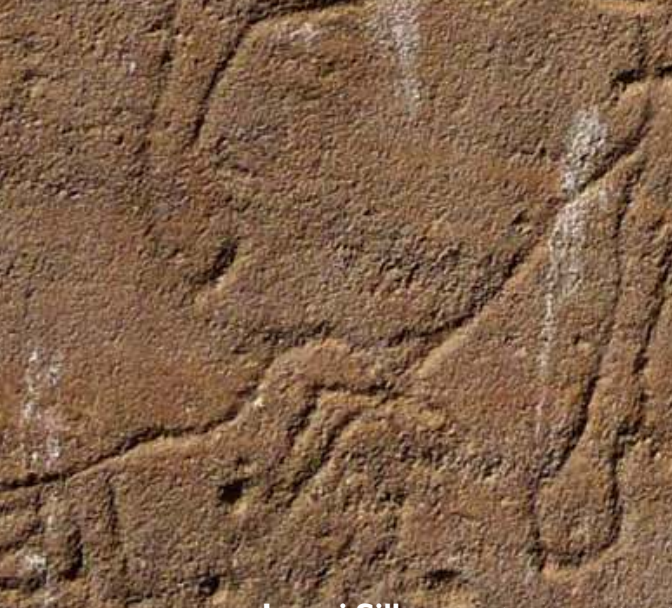

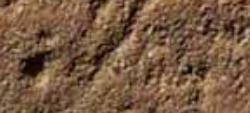

(1)

\section{Le roi silko en}

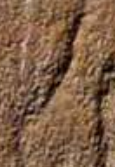


comme celui de «tous les Éthiopiens», c'est-à-dire des différents peuples qui constituent au ve siècle la population de Nubie, incluant les Méroïtes désormais assujettis à la nouvelle élite et sans doute, au terme de ses combats victorieux, les Blemmyes. Néanmoins, le titre par lequel il se désigne, en grec basiliskos, "petit roi", ménage la suzeraineté au moins théorique du basileus, titre de l'empereur romain, et suggère que Silko était un allié ( foederatus) de Rome. Il semble d'ailleurs que le nom même de Silko soit construit sur le mot protonubien *sil qui désigne un chef suprême et s'est conservé jusqu'à nos jours avec le sens de «roi» en nubien du Kordofan (šil). Si l'élément final $k o$ est bien, comme on peut le supposer, le groupe méroïtique qo, «celui-ci est ", largement attesté dans les noms royaux koushites (Taharqo, Aramatelqo, Tabirqo, etc.), l'ensemble serait un composé mixte nubio-méroïtique emblématique du roi «de tous les Éthiopiens» probablement adopté comme nom de couronnement, plutôt qu'un nom de naissance.

Ses guerres contre les Blemmyes sont le sujet principal du texte, puisqu'il s'agit de montrer en ce temple de Kalabcha, jusqu'alors capitale religieuse de ce peuple en Basse-Nubie, qu'il est désormais le maitre des lieux. Cette victoire s'est construite sur trois campagnes, la première ayant été conclue par un traité de paix que les Blemmyes n’ont pas respecté et la dernière par l'occupation de leur cité principale par les armes. Il semble toutefois que les guerres menées par Silko ne mirent pas un terme aux ambitions des Blemmyes puisque son successeur Abourni se vit réclamer les territoires confisqués, comme nous le verrons plus loin.

Silko fut très probablement inhumé dans la nécropole royale

des origines

à la chute

du sultanat

Fung de Ballaña, récemment inaugurée, qui remplaçait le cimetière de Qustul, situé sur l'autre rive, où quatre générations de chefs noubades avaient été enterrées sous des tumuli depuis environ 370 ou peut-être un peu auparavant. Découvertes par Walter Emery en 1928, les sépultures de la nouvelle nécropole étaient à la mesure du pouvoir croissant des Noubades. Elles étaient couvertes de larges tumuli dont les plus importants dépassent $70 \mathrm{~m}$ de diamètre et $13 \mathrm{~m}$ de hauteur. Les défunts y reposaient sur des angarebs (lits de bois soudanais) dans des appartements en briques crues, au milieu de trésors de provenances diverses. Y figuraient notamment des chaises curules, tabourets pliants réservés aux magistrats à imperium dans la Rome antique: des cadeaux diplomatiques accompagnant les traités et qui, ici, témoignaient clairement du statut d'alliés que les Romains avaient concédé aux rois noubades. Furent aussi retrouvées, parfois encore fixées sur le crâne des souverains, des couronnes en argent d'inspiration méroïtique, également influencées par l'orfevrerie byzantine, où des pierres fines en cabochon voisinaient avec des yeux oudjat et des cobras royaux: d'autres symboles de leur pouvoir, mais cette fois-ci de fabrication locale. 
Le plus frappant pour les fouilleurs fut toutefois le grand nombre d'animaux et surtout d'humains sacrifiés qui, pour longtemps, fit passer les Noubades pour des Barbares. Toutefois, on est assez loin des chiffres des tumuli de Kerma (voir chapitre 3, p. 59 sq.) : à Qustul, on dénombre au maximum 19 personnes par tombe et 9 à Ballaña. Il ne s'agit pas à proprement parler de sacrifices humains, encore moins de massacres rituels d'ennemis, comme le proposait l'archéologue Patrice Lenoble qui interprétait de façon trop littérale les représentations de souverains koushites abattant des prisonniers. Il s'agit en fait d'un thème emprunté à l'Égypte, où il n'avait pas d'autre réalité que de symboliser en un raccourci iconographique les victoires militaires de Pharaon. Les victimes des tumuli noubades sont clairement des «morts d'accompagnement», les serviteurs du souverain ayant été mis à mort pour qu'ils continuent leurs fonctions auprès de lui dans l'Au-delà. Cette coutume avait disparu à Koush entre la fin du royaume de Kerma et la phase ancienne du royaume de Méroé, peut-être sous l'influence de l'Égypte où elle avait été abandonnée dès la II ${ }^{e}$ dynastie. Mais elle est à nouveau attestée, quoiqu'en très petits effectifs, à partir de la période méroïtique classique. Néanmoins, plutôt que d'y voir une preuve supplémentaire de la continuité des usages postméroïtiques avec le royaume de Méroé, il faut plutôt penser à une tradition commune aux Noubades, que l'on retrouve dans d'autres cimetières princiers comme Firka, au nord de l'île de Saï. En revanche, les nécropoles situées plus au sud, comme Tanqasi ou el-Hobagi, attribuables à d'autres groupes de Noubas, n'ont pas livré de morts d'accompagnement.

Si Kalabcha fut l'éphémère capitale des Blemmyes sur le Nil, il semble que les souverains noubades, bien qu'inhumés à Ballaña, un peu en amont de la deuxième cataracte, aient fixé leur résidence à Qasr Ibrim, une ville fortifiée située à $70 \mathrm{~km}$ au nord. Cette cité bâtie en hauteur sur un rocher a par chance échappé à l'immersion dans le lac de retenue du barrage d'Assouan et a livré aux fouilleurs de l'Egypt Exploration Society des documents historiques de première importance en de nombreuses langues, du démotique à l'arabe, en passant par le méroïtique, le grec, le copte et le vieux-nubien. En 1976, un lot d'archives contenant quatre lettres en grec et en copte a été mis au jour. La plus longue est l'original d'un message adressé par le roi blemmye Phonen au roi des Noubades Abourni, successeur de Silko. Bien qu'écrite dans un sabir grec difficilement intelligible, elle a fait l'objet en 1998 dans les Fontes Historiae Nubiorum d'une nouvelle édition par Richard Holton-Pierce, qui en améliore grandement la compréhension.

Phonen commence par saluer fort courtoisement Abourni et ses deux fils, Nakasé et Mousês. Il rappelle qu'il est issu d'une noble lignée et cite son propre fils, Breitek, ainsi que ses frères. Le sujet principal 
de la lettre est la question de la restitution des terres confisquées par Silko, comprenant notamment la cité de Talmis (Kalabcha): «Silko le premier a vaincu et pris Talmis. Aujourd'hui, tu as vaincu et pris Talmis. Silko le premier nous a pris et interdit nos terres." Le roi noubade avait alors exigé une rançon de bovins, de petit bétail et de chameaux pour redonner aux Blemmyes leurs terres conquises. Or, Phonen avait livré ces animaux sans que pour autant elles lui eussent été rendues. Il rappelle donc ce pacte à Abourni, qui semble avoir récemment succédé à Silko. D'autres demandes de restitution accompagnent cette requête: les statues des dieux adorés par les Blemmyes auraient été elles aussi confisquées. Qui plus est, un des frères de Phonen, Yeni, a été tué par les soldats d'Abourni alors qu'il avait été envoyé en ambassade; malgré la maladresse du texte grec (thanatos «la mort" pris pour un adjectif), il est vraisemblable que c'est son corps que réclame Phonen. De façon générale, malgré ses manifestations de fierté et même quelques menaces voilées, la lettre de Phonen présente le roi blemmye dans une position pitoyable: défait une première fois et floué par Silko, vaincu à nouveau par Abourni, il n'a clairement pas les moyens militaires de renverser la situation et invoque en désespoir de cause la parole donnée, alors que son peuple a notoirement bafoué tous les traités de paix dans le passé. On ignore la réponse d'Abourni, mais il est certain que les Blemmyes ne récupérèrent jamais Kalabcha, où ils n’auraient pas manqué en ce cas d'effacer l'humiliante inscription triomphale de Silko.

Les trois autres lettres retrouvées à Qasr Ibrim dans le même lot que celle de Phonen sont en copte et adressées au chef local (phylarkhos) des origines à la chute du sultanat Fung de la "nation des Anouba", c'est-à-dire au gouverneur noubade de la région limitrophe de la frontière égyptienne, qui répondait au nom de Tantani. Une première a été envoyée par un certain Viventius, commandant de l'armée romaine postée de l'autre côté de cette frontière. Elle traite de l'établissement d'un traité de paix entre Noubades et Romains, pour lequel une rencontre entre les deux officiels est envisagée. Il pourrait en fait s'agir d'un renouvellement de l'alliance que Silko avait très probablement conclue avec ses voisins et qui transparaît dans son inscription triomphale de Kalabcha. On se souvient que l'historien grec Procope de Césarée, qui écrivait au milieu du vi ${ }^{e}$ siècle, faisait remonter à 298 , c'est-àdire au retrait des armées romaines de la Basse-Nubie, un traité par lequel le Dodécaschène avait été cédé aux Noubades. À cette date, comme l'ont remarqué tous les spécialistes, le royaume de Méroé était encore maitre de la région, et on a plutôt envisagé qu'il fût le vrai bénéficiaire de cette dévolution. Toutefois, il est également possible que Procope ait simplement été abusé par des sources inexactes sur la chronologie des événements et qu'un traité au profit des Noubades ait été effectivement négocié, 
leur reconnaissant entière suzeraineté sur la Basse-Nubie au détriment des Blemmyes. Mais il aurait été conclu au ve siècle, lorsque ce peuple a pu être considéré par les Romains comme un rempart fiable contre les Blemmyes. Le règne de Silko est alors le moment le plus probable et expliquerait l'assurance du monarque face à ses ennemis.

Une seconde lettre adressée à Tantani provient d'un certain Iahatek, dont le nom est clairement blemmye (tek signifie "homme» et termine fréquemment les anthroponymes). Elle est à ce point fragmentaire qu'il est impossible de comprendre son objet. Le rédacteur demande d'envoyer quelque chose à Talmis (Kalabcha), ce qui prouve que certains Blemmyes y étaient encore présents sous le règne d'Abourni, bien que soumis aux Noubades, et qu'ils n'en avaient pas été tous expulsés, comme s'en plaignait le roi Phonen.

\section{L'influence croissante du christianisme dans la Nubie postméroïtique}

La troisième lettre en copte retrouvée dans le lot d'archives noubades de Qasr Ibrim a été expédiée par un moine de Philae, dénommé Mousês (Moïse). Elle concerne toutefois des préoccupations bien temporelles, à savoir l'envoi depuis l'Égypte de produits de luxe, id est de la pourpre et du poivre. Sa formule finale, «salut par le Christ, frère bien-aimé», semble désigner Tantani comme un de ses coreligionnaires. Tantani n'aurait en ce cas pas changé son nom païen pour un nom chrétien, mais on connaît des exemples où une personne convertie est désignée par ses deux noms: ainsi dans des documents d'une enclave blemmye à Gébelein, en Haute-Égypte, au siècle suivant, une femme est appelée "Amnas, dont le nom chrétien est Sophia". D'autre part, les premiers rois de Nobadia connus comme chrétiens, au $\mathrm{VI}^{\mathrm{e}}$ siècle, Eirpanomé et Tokiltoéton, portent encore des noms nubiens. Des conversions individuelles au christianisme sont attestées sporadiquement chez les Noubades dès le $v^{e}$ siècle. Nous avons vu précédemment, dans la lettre de Phonen, qu'un des fils du roi Abourni s'appelait Mousês, comme le moine de Philae, correspondant de Tantani. Des Égyptiens chrétiens étaient par ailleurs employés à la cour royale, comme vraisemblablement le scribe qui a rédigé l'inscription triomphale du roi Silko.

La Nubie ne pouvait en effet rester isolée des profonds changements religieux qui s'opéraient au nord de sa frontière. En 380, l'empereur Théodose Ir avait décrété, par l'édit de Thessalonique, que le christianisme catholique serait la seule religion permise dans l'Empire romain. Toutefois, cette décision fut difficile à appliquer à la lettre. Ainsi, les sanctuaires païens en bordure de frontières bénéficièrent de tolérances. Tel fut le cas 
le Soudan

\section{2}

des origines

à la chute

du sultanat

Fung des temples de Philae, situés dans une enclave romaine à l'intérieur du territoire nubien, puisqu'aussi bien les Méroïtes que les Noubades et les Blemmyes continuaient à rendre un culte à ses divinités, en premier lieu Isis dont la statue en bois était annuellement acheminée dans sa barque sacrée jusqu'aux temples de Basse-Nubie. Le fonctionnement de Philae était donc un enjeu diplomatique. Les Romains en usèrent par exemple en 452 , lorsqu'ils tentèrent d'établir un traité de paix avec les Blemmyes et les Noubades, ainsi que nous l'avons mentionné précédemment, en leur concédant un droit d'accès aux sanctuaires de Philae.

Ce n'est peut-être pas un hasard si le dernier texte égyptien, un graffito démotique commémorant la participation des frères Smet l'Aîné et Smet le Jeune, tous deux prêtres d'Isis, aux fêtes de Khoïak en l'honneur d'Isis et d'Osiris, a été gravé justement en cette année 452 . D'après Jitse Dijkstra, spécialiste de l'Antiquité tardive en Égypte, cette famille aurait fourni les derniers représentants du clergé d'Isis de Philae, vivant en autarcie dans un environnement presque entièrement christianisé. Il n'est d'ailleurs pas impossible qu'ils aient été Méroïtes, car on possède d'eux des graffiti grecs et démotiques (les derniers de Philae), mais trois inscriptions méroïtiques très tardives citent des «Smet» à proximité des précédentes. Dans ce cas, elles constitueraient également les derniers textes écrits dans la langue de Koush, plusieurs décennies après l'inscription du roi blemmye Kharamadoyé à Kalabcha.

Ce dernier bastion du paganisme, nous rapporte l'historien Procope, aurait été balayé vers 537, lorsque l'empereur Justinien décida d'envoyer le général Narsès, qui commandait les troupes dans la région, démolir les sanctuaires, arrêter les prêtres et envoyer les statues divines à Constantinople. Toutefois, comme le remarque Jitse Dijkstra, les temples ne furent pas détruits, et il est probable qu'ils n'abritaient plus aucune activité cultuelle continue depuis près de quatre-vingts ans. Mais c'est sans doute à ce moment que le sanctuaire d'Isis fut transformé en église consacrée à saint Étienne et placée sous la direction de l'évêque Théodore. Plusieurs inscriptions en grec gravées sur ses murs commémorent cette conversion de l'édifice. La plus brève scelle cruellement la défaite du monde ancien: «La Croix a vaincu. Elle vainc toujours.» 